\title{
SiRNA in MSCs-Derived Exosomes Silences Ctgf Gene for Corticospinal Axon Regeneration and Locomotor Recovery in Spinal Cord Injury Rats
}

\section{Wei Huang}

Affiliated Zhongshan Hospital of Dalian University

\section{Mingjia Qu}

Affiliated Zhongshan Hospital of Dalian University

Lu Li

Affiliated Zhongshan Hospital of Dalian University

Tao Liu

Affiliated Zhongshan Hospital of Dalian University

\section{Ruimeng Duan}

Affiliated Zhongshan Hospital of Dalian University

\section{Miaoman Lin}

Affiliated Zhongshan Hospital of Dalian University

Yashuai Yuan

Affiliated Zhongshan Hospital of Dalian University

\section{Meng Zhang}

Affiliated Zhongshan Hospital of Dalian University

Junxiao Gao

Affiliated Zhongshan Hospital of Dalian University

Xiaobing Yu ( $\square$ yuxiaobing1976@hotmail.com )

Affiliated Zhongshan Hospital of Dalian University

\section{Research}

Keywords: CTGF, spinal cord injury, exosome, small interfering RNA, electroporation, RNA delivery

Posted Date: December 10th, 2019

DOI: https://doi.org/10.21203/rs.2.18408/v1

License: (c) (1) This work is licensed under a Creative Commons Attribution 4.0 International License.

Read Full License 


\section{Abstract}

As RNA interference (RNAi) received solicitous attention by many for its promising performance in gene therapy recently, and how to obtain a choice small interfering RNA (siRNA) vector has become a moot point at this moment. Exosomes (Exo) show advantages of long survival time in vivo, high transmission efficiency and easy penetration across the blood-brain/spinal cord barrier, renowned as excellent carriers of bioactive substances. Previous studies have confirmed the detrimental effect of connective tissue growth factor (CTGF) on axonal regeneration after spinal cord injury (SCl) via stimulating the unrestricted growth of glial scars. In this study, we applied mesenchymal stem cells (MSCs)-derived Exo as the delivery of synthesized siRNA, which were extracted from rat bone marrow. We constructed exosome-siRNA (Exo-siRNA) that could specifically silence Ctgf gene in the injury sites by electroporation.

During the administration, we injected Exo-siRNAs into the tail vein of $\mathrm{SCl}$ rats, and their distribution and accumulation in the spinal cord were visualized by in vivo fluorescence imaging. Relevant in vivo and in vitro experiments in this study showed that Exo-siRNA not only effectively inhibited the expressions of Ctgf gene and glial scar formation related proteins, such as GFAP, vimentin fibronectin and laminin, in the injured spinal cord segments, but quenched inflammation, and thwarted neuronal apoptosis and reactive astrocytes and glial scar formation. Besides, it significantly up-regulated several neurotrophic factors and anti-inflammatory factors (e.g., TGF- $\beta 1$ ), acting as a facilitator of axon regeneration of nerve-injured motor neurons. Thus, the motor function of $\mathrm{SCl}$ rats were remarkably promoted.

In conclusion, this study has combined the thoroughness of gene therapy and the excellent drug-loading characteristics of Exo for the precise treatment of $\mathrm{SCl}$, which will shed new light on the drug-loading field of Exo.

\section{Introduction}

$\mathrm{SCl}$ is one of the most serious complications of spinal fracture, which mostly results in severe sensory and motor dysfunction, even paraplegia. The regeneration of damaged corticospinal axons is a critical issue that challenges doctors for years. ${ }^{1}$ The World Health Organization has stated a rate of 40 cases of traumatic SCI per million people worldwide every year, of which up to $50 \%$ can cause varying degrees of quadriplegia. ${ }^{2} \mathrm{MSC} s$ with a strong potential in proliferation and differentiation has made great strides in the treatment of $\mathrm{SCl}^{3}$ featuring significant recovery of motor dysfunction in $\mathrm{SCl}$ rats. However, problems such as teratogenesis, immune rejection, pulmonary embolism, low transplantation rate and survival rate inevitably pose challenges to most researchers. ${ }^{4}$ Recent studies have explained that the Exo secreted from MSCs may take the central role behind their potential therapeutic effect on SCl. ${ }^{5}$ Moreover, apart from Exo's penetration across the blood-brain/spinal cord barrier depending on their small size, they also manifest a promising advantage of homing to injury sites of the central nervous system driven by inflammation. ${ }^{6,7}$ Their specific targeting provides the feasibility for drug delivery to the injured spinal cord segments. 
Exo is nano-sized vesicles with the diameter of $30-200 \mathrm{~nm}$ secreted by various cells. ${ }^{8}$ As an important means of cell-to-cell interaction, Exo can enter target cells via receptors-mediated endocytosis, transmitting the messenger RNA (mRNA), small non-coding RNA, protein and so forth to participate in the regulation of biological information. ${ }^{9-11}$ Numerous studies across the world have shown that intravenous injection of MSCs-derived Exo is more effective than other administrations of MSCs in repairing traumatic brain injury, stroke and other central nervous system injuries. ${ }^{12}$ In the experiment of central nervous system injury, MSCs-derived Exo have facilitated the growth of axons of cortical neurons. ${ }^{13}$ In stroke rats, MSCs-derived Exo have been systemically administered to promote neurovascular remodeling and restore neurological function. ${ }^{14,15}$ In addition, Exo derived from human umbilical cord MSCs also ameliorate the inflammatory microenvironment following $\mathrm{SCl}$ by down-regulating expressions of inflammatory factors, such as TNF- $a$, MIP- $1 a$, IL- 6 and IFN- $y .{ }^{16}$ The gene therapy based on stem cell-derived Exo increases medication safety, so MSCs-derived Exo as a commensurate alternative therapy can be expected for stem cell therapy. ${ }^{17}$

CTGF is a secretory protein with multiple regulatory functions, which plays important roles in the physiological processes of bronchial and lung formation and pathogenesis of respiratory diseases, vascular atherosclerosis and renal fibrosis. ${ }^{18,19}$ CTGF as a growth regulator of glial scar is essential in axon regeneration after $\mathrm{SCl}^{20} \mathrm{Glial}$ scar is a chronic, physical and chemical barrier that inhibits axon regeneration of the impaired neurons in the spinal cord in mammals. ${ }^{21}$ After $\mathrm{SCl}, \mathrm{CTGF}$ is involved in initial activation of glial cells in the injury sites. Specifically, the overexpression of CTGF accelerates the proliferation of glial scars, thwarting axon migration and regeneration. ${ }^{22}$ However, conditional deletion of CTGF significantly reverses the effect, reducing glial scar proliferation. Adenovirus- or lentiviral-mediated RNAi is the mainstream method currently used as a RNA knockout technology, which can continuously silence specific genes by introducing target siRNA into cells and continuously express it through virus infection. ${ }^{23,24,25,26}$ Despite the strong infectivity and integration of viral vectors, these exogenous viruses can easily interfere with the expressions of host genes with carcinogenic risks. Therefore, the preoccupation is to find a safer and more efficient siRNA delivery vector for clinical practice. Exo as natural vesicles secreted by autologous cells present low immune rejection and can transmit biological genetic information through blood-brain barrier. So they can be regarded as an ideal siRNA vector. Shaowei Guo et al. confirmed that loading phosphatase and tensin homolog (PTEN) siRNA with MSC exosomes significantly promoted the growth of axons and neovascularization following $\mathrm{SCl}$, and significantly facilitated the rehabilitation of SCI patients. ${ }^{27}$

In the study of how to load siRNA into Exo, electroporation has been proved to be a good way. Faruqu Farid et al. succeeded in loading ATOT655-siRNA into human embryonic kidney (HEK)-293-derived Exo by electroporation, ${ }^{28}$ and Aqil Farrukh et al. successfully introduced siRNA targeting v-Ki-ras2 Kirsten rat sarcoma viral oncogene homolog (KRAS) gene into milk-derived Exo by the same method, showing good loading rate and silencing efficiency. ${ }^{29}$ 
In this study, we designed and synthesized three pairs of siRNAs targeting Ctgf gene, screened out the pair with the highest silencing efficiency, effectively transfected the selected siRNA into Exo by electroporation, and explored the optimal voltage, resistance and capacitance of transfection efficiency. In this way, we successfully constructed Exo-siRNA. In addition, our in vitro and in vivo experiments proved that Exo effectively transported siRNA to the impaired segments of spinal cord and significantly inhibited the expression of Ctgf gene. The Exo-siRNA also improved the micro-environment of injured spinal cord, inhibited inflammation and neuron apoptosis, reduced glial scars formation, facilitated axon regeneration, and promoted motor function recovery in $\mathrm{SCl}$ rats (Figure 1).

\section{Results And Discussion}

\section{Characterization of MCSs-Exo and Exo-siRNAs}

As was mentioned above, ${ }^{29}$ Exo were separated by differential centrifugation and density gradient centrifugation, and the supernatant containing affluent MSCs was extracted from the bone marrow of Sprague-Dawley (SD) rats. Characterization of Exo and the corresponding Exo-siRNAs were preliminarily compared. Transmission electron microscopy (TEM) revealed that the typical saucer-like morphology with the diameter of 30-200 nm was found in both Exo and Exo-siRNAs (Figure 2a). Nanoparticle tracking analysis (NTA) also showed that most Exo and Exo-siRNAs were around $150 \mathrm{~nm}$ in diameter with little difference, indicating that the incorporation of siRNA did not affect the shape and diameter of Exo (Figure 2b). Results of western blotting (WB) suggested that the expressions of exosome markers, CD9 and CD63, were impervious to siRNA loading (Figure 2c).

Figure 2: Characterization of Exo and Exo-siRNA. (a) TEM revealed the typical exosome structure of Exo and Exo-siRNA. (b) NTA analysis of Exo and Exo-siRNA showed the incorporation of siRNA did not affect the diameter of Exo. (c) The exosome marker proteins, CD9 and CD63, were analyzed by WB. (d) Exo stained by fluorescence of DilC $_{18}$ amethylindocarbocyanine were exhibited, scale bar $=100 \mu \mathrm{m}$.

\section{Screening of siRNA and preliminary verification of its silencing efficiency}

In the following experiment, we designed and synthesized three pairs of siRNAs (namely, siRNA-1, 2 and 3凶targeting Ctgf gene, and labeled them with Cy3. The gene sequences of the three siRNAs were listed in Table 1. Their silencing efficiency was estimated. We transfected HEK293T cells with siRNA-1, 2 and 3 by Lipofectamine $^{\text {TM }} 3000$ reagent (Figure 3a), and verified by RT-PCR. The results showed that siRNA-1, 2 and 3 inhibited the expression of Ctgf gene to a certain extent, ranging from $18.85 \pm 7.75 \%$ to $65.52 \pm 7.52 \%$. As a result, siRNA-2 presented stronger inhibitory effect than the other two (Figure 3b), which was preferable to be loaded on the Exo.

\section{The MSCs-Exo presented high loading rate of siRNA with high stability}


We loaded siRNA-2 into MSCs-Exo by electroporation. The EC630 (BTX, USA) electroporation system was adopted at 8 different conditions with changes in voltag, resistance and capacitance (Table 2). The loading process of the 8 groups was conducted in a pulse time of 10-15 ms. According to the reference system,28 $150 \mu \mathrm{L}$ reaction solution contained $500 \mathrm{nM}$ siRNA and $7 \mu \mathrm{g}$ Exo, and the rest was filled with electroporation buffer. Among the 8 groups, the reaction solution of Group 7 encompassed $21 \mu \mathrm{g}$ Exo and $10 \mu \mathrm{L}$ siRNA, and that of Group $821 \mu \mathrm{g}$ Exo and $20 \mu \mathrm{L}$ siRNA. When electroporation was accomplished, Exo were extracted from electroporation buffer and siRNA extraction, so Exo-siRNAs were obtained. Absolute quantitative Real-Time PCR results showed that Exo of the 8 groups were loaded with a certain amount of siRNAs. The loading efficiency of siRNA was the highest (Figure 3c) in Group 2, indicating that its electroporation system was optimum. The count of siRNA nanoparticles per microgram of exosome was the highest in Group 2, reaching about $5.08 \times 10^{11} / \mu \mathrm{g}$.

Combining results of Table 2 and Figure 4a, it was found that the lower the capacitance, the more siRNA molecules were loaded into Exo under the same voltage and resistance conditions in Group 1 to 4 . In comparison with Group 7 and 8, Group 8 presented fewer number of siRNA molecules loaded into Exo, with more siRNAs remaining in the reaction solution, than Group 7 under the same voltage, resistance and capacitance conditions. This indicated that the same electroporation condition often corresponded to an optimum concentration of siRNAs. Group 8 was the control group without electric shock, but a certain amount of siRNA could still be detected. This can be explained by the non-specific binding of siRNA to Exo. Later, we applied 5'-32P-labeled siRNA to measure siRNA loading rates in Exo. Radioactivity in the pellet and supernatant was determined by a known volume to a PEl-cellulose layer and quantitated by Packard Instantlmager. The loading rate was calculated by the following formula: siRNA loading = (CPM in pellet / CPM in supernatant $) \times 100$. Finally, the siRNA loading rates of the 8 groups were obtained, which were in line with the corresponding RT-PCR results. Group 2 achieved the highest siRNA loading rate / electroporation efficiency, of about 33.24\% (Figure 3d). So the optimal parameters for electroporation system are as follows: voltage, $40 \mathrm{v}$; resistance, $50 \Omega$; capacitor, $500 \mu \mathrm{F}$; exosome volume, $7 \mu \mathrm{L}$; and siRNA volume, $3.75 \mu \mathrm{L}$.

In order to test the stability / anti-degradation ability of Exo-siRNA, we cultured the siRNAs delivered by blood-derived Exo with RNase A ( $0.01 \mu \mathrm{g} / \mathrm{mL}$, simulating body fluid environment) for 45 min and naked siRNA as the control group for $45 \mathrm{~min}$. The relative content of siRNA was determined by RT-PCR. As a result, the content of siRNA in exogenous siRNA was significantly higher than that of naked siRNA. After 45-min RNase A treatment, 75.1\% siRNA residues remained in the exogenous siRNA group, compared with less than $50 \%$ in the naked siRNA group. This indicated that Exo as natural carriers of exogenous siRNA showed favorable encapsulation ability and effectively prevented the degradation of siRNA in body fluids. They could be renowned as ideal siRNA carriers (Figure 3e).

In vitro study of Exo-siRNA as a treatment for SCl

Exo-siRNA effectively inhibited the expression of Ctgf gene in astrocytes and stimulated the secretion of neurotrophic factors and anti-inflammatory factors in the impaired spinal cord. 
PKH67 is a green fluorescent dye that specifically binds to lipid bilayers. In this experiment, we adopted it for labeling Exo and the optimal electroporation system for effectively obtaining high amount of doublelabeled Exo-siRNAs (PKH67 for Exo and Cy3 for siRNAs) for the subsequent cell and animal experiments.

Here we investigated the underlying effect of Exo-siRNAs on SCl. Rat astrocytes were treated with lipopolysaccharide (LPS) for simulating the environment of $\mathrm{SCl}$, and then cultured under hypoxia $\left(2 \% \mathrm{O}_{2}\right)$ for 24 h. Subsequently, Exo-siRNA was co-cultured with rat astrocytes. Conventional transfection using Lipo3000 transfection reagent was used as the control group. Astrocytes of the control group were incubated with PBS (LPS/Hypoxia), while those of other three groups were administered with siRNA (LPS/Hypoxia + siRNA), Exo (LPS/Hypoxia + Exo) and Exo-siRNA (LPS/Hypoxia + Exo-siRNA). After 24-h administrations, confocal fluorescence microscopy revealed that the cells treated with Exo-siRNA were double-positive for green fluorescence (Exo) and red fluorescence (siRNAs) in Figure 4a. RT-PCR and WB uncovered that both Exo-siRNA group and the control group significantly down-regulated the expression of $\operatorname{Ctgf}$ gene, with the down-regulatory rates of $21 \%$ and $19 \%$, respectively (Figure $4 \mathrm{~b}$ ). This indicated that Exo were competent in delivering siRNA for interfering target genes. Besides, RT-PCR results showed that Exo-siRNA facilitated secretions of neurotrophic factors (e.g., BDNF) and anti-inflammatory factors (e.g., TGF- $\beta 1$ ) by astrocytes, which was undoubtedly of therapeutic significance of $\mathrm{SCl}$ (Figure 4c).

\section{Exo-siRNA thwarted neuronal apoptosis in the impaired spinal cord segments}

A previous study reported inhibitory effect of MSCs-Exo, acting as neural protectors, on neuronal apoptosis and inflammatory factors. ${ }^{30}$ In this study, Exo-siRNA was co-cultured with LPS and dorsal root ganglion cells (DRGs) of hypoxia-treated rats for $24 \mathrm{~h}$. The DRGs in the control group were exempt from the administration of LPS/Hypoxia. The remaining four groups were SCl group (LPS/Hypoxia + PBS), siRNA treatment group (LPS/Hypoxia + siRNA), Exo treatment group (LPS/Hypoxia + Exo) and Exo-siRNA treatment group (LPS/Hypoxia + Exo-siRNA). After the 24-h administrations, cell viability was assessed by living and death staining (Figure $5 a$ ) and quantitative analysis of CCK-8 (Figure $5 \mathrm{~b}$ ). It was found that the survival rate of DRGs in $\mathrm{SCl}$ group was significantly reduced by less than $50 \%$. Exo and Exo-siRNA treatment groups showed higher survival rates of DRGs than SCI group, which were close to $90.0 \%$. There was nonsignificant difference between the two groups, indicating that the anti-apoptotic effect of ExosiRNA mainly attributed to Exo.

\section{Exo-siRNA propelled the balance of macrophages towards $\mathrm{M} 2$ in $\mathrm{SCl}$ environment and quenched inflammation}

According to previous researches, MSCs-Exo can induce macrophage polarization and inhibit inflammation in SCl. ${ }^{30-32}$ Here we explored the promoting effect of Exo-siRNA on macrophage polarization. NR8383 cells derived from rat alveolar macrophages were cultured with LPS/hypoxia for 24 $\mathrm{h}$ and then transformed into M1 state. After 48-hours incubation of siRNA, MSC-Exo and Exo-siRNA, the expressions of $\mathrm{M} 1$ and $\mathrm{M} 2$ markers were quantitated by qRT-PCR and an ELISA assay. The results 
showed that the expressions of M1-related pro-inflammatory cytokines, TNF- $a$ and IL-6, significantly dropped in Exo or Exo-siRNA treatment groups with nonsignificant differences between the two groups (Figure 5c). Administrations of Exo and Exo-siRNA significantly boosted mRNA expressions of M2 macrophage markers, Arg-1 and CD206, without significant difference between the two groups. The expressions of anti-inflammatory factors, IL-4 and IL-10, in M2 macrophages were remarkably elevated in Exo and Exo-siRNA treatment groups (Figure 5d). However, these changes were not evident in siRNA treatment group, which indicated that siRNA had no direct regulatory effect on inflammatory factors.

\section{In vivo study of Exo-siRNA in the treatment of SCI}

\section{Exo-siRNA passed through the blood/spinal cord barrier and accumulated at the impaired sites of SCI}

We further investigated the therapeutic effect of Exo-siRNA on SCl rats. They were divided into three groups: SCl group (PBS treatment), Exo group (pure exosome treatment) and Exo-siRNA group (Exo-siRNA treatment). Exo-siRNA was administered to $\mathrm{SCl}$ rats by intravenous tail vein injection, and its targeting to spinal cord segments was traced by living animal fluorescence imaging system. Reference imaging required a specific wavelength range. DiR iodide (a red lipophilic fluorescent dye) was selected as the fluorescent labeling dye for Exo and Exo-siRNA. The main organs, such as heart, lung, liver, kidney and spleen, and spinal cord were harvested at $3 \mathrm{~h}, 24 \mathrm{~h}$ after injection and examined by fluorescence imaging system. A majority of Exo and Exo-siRNA accumulated in the liver and bilateral lungs (Figure 6a). It was found that Exo and Exo-siRNA presented relatively high fluorescence intensity in the liver based on the 3-h imaging, which was consistent with previous studies. Exo and Exo-siRNA were mainly absorbed by mononuclear phagocytes in the liver after systemic injection, and there was fluorescence in the spinal cord, indicating chemotactic activity for Exo and Exo-siRNA in the injured spinal cord segments. The fluorescence intensity of all organs decreased dramatically $24 \mathrm{~h}$ after injection. Exo and Exo-siRNA were absorbed by various nerve cells in the liver and injured spinal cord.

\section{Exo-siRNA significantly decreased the expression of Ctgf gene, inhibited the expressions of GFAP, vimentin, fibronectin and laminin, and repressed glial scar formation}

To verify the silencing effect of Exo-siRNA in vivo, $\mathrm{SCl}$ rats received intravenous injection of Exo and ExosiRNA for 5 consecutive days from the date of modeling, and the rats in sham operation group and control group were administered with the same volume of saline. At the $4^{\text {th }}$ week, the damaged segments was sampled, Protein were extracted for WB analyses. Compared with SCl rats, Exo-siRNA-treated rats showed a $41.2 \%$ decrease in the corresponding protein level (Figure 6b). However, there was no evidence that Exo could also regulate CTGF. It has been proved that CTGF can down-regulate the expressions of proteins related to glial scar formation, including GFAP, vimentin, fibronectin and laminin. ${ }^{24}$ The four proteins were determined by WB in this study. As a result, the four were down-regulated in both Exo and Exo-siRNA groups compared with $\mathrm{SCl}$ group. The down-regulatory rate of GFAP was $55.7 \%$, and that of vimentin was $44.6 \%$, fibronectin $52.2 \%$ and laminin $25.5 \%$ in Exo-siRNA group; and GFAP $25.8 \%$, vimentin $14.5 \%$, fibronectin $12.1 \%$ and laminin $12.9 \%$ in Exo group. Moreover, the injured spinal cord tissue of each 
group was collected at the $4^{\text {th }}$ week. The formation of glial scar was measured by Masson staining (Figure 6c), and the area of glial scar was calculated by Image $\mathrm{J}$ software (V1.8.0.112). The results showed that the affected spinal cord segments by glial scar in Exo-siRNA group was 65.6\%, less than the figure in $\mathrm{SCl}$ group. The affected area of glial scar in Exo group was 24.4\%, less than that in SCI group . These results indicated that Exo repressed the formation of glial scar. However, the underlying mechanism remains unclear. $5,33,34$

\section{Exo-siRNA ameliorated motor dysfunction of SCl rats}

To investigate whether Exo-siRNA can restore SCl-induced motor dysfunction, Basso, Beattie and Bresnahan (BBB) locomotor scale were adopted to evaluate the motor activity of rats in each group at 1 , $7,14,21,28,35,42$ and 48 days after injury. The measurement lasted $4 \mathrm{~min}$. The BBB scores of all groups increased gradually with time, which indicated that rats had a certain ability of self-recovery. On the $48^{\text {th }}$ day, Exo and Exo-siRNA groups exhibited significant functional recovery of locomotor activity compared with $\mathrm{SCl}$ group. The average score of rats in Exo-siRNA group was $15.4+1.5$ points, and that in Exo group was $10.8+0.5$ points. Rats in Exo-siRNA group showed better functional recovery than those in Exo group, which was consistent with and could be explained by corticospinal axon regeneration in the two groups (Figure 6d).

\section{Exo-siRNA promoted corticospinal axon regeneration}

Four weeks after modeling, the longitudinal sections of spinal cord tissues of each group were sampled. The extent of neuronal injury and glial scar formation were studied using double-labeled immunofluorescence with neurofilament (NF-H) and glial fibrillary acidic protein (GFAP) as the corresponding markers. In SCl group, astrocytes were recruited and neurons were disintegrated compared with the control group. On the contrary, Exo and Exo-siRNA groups showed significant decreased expressions of astrocytes and increased levels of neurofilaments (Figure 7a) compared with SCl group. Due to the silencing effect of siRNA on Ctgf gene, Exo-siRNA group exhibited lower count of glial scars and higher count of neurofilaments than Exo group. These indicated that Exo-siRNA promoted corticospinal axon regeneration in the impaired spinal cord segments.

\section{Materials And Methods}

\section{Preparation of MSCs and isolation of Exo}

Two 2-week-old SD female rats, purchased from Animal Experimental Center of Dalian Medical University, were regularly housed. As was mentioned above, ${ }^{5}$ bone marrow MSCs were extracted by whole bone marrow culture method and cultivated in primary culture. When the cell density reached $80 \%$, expanded 
culture was carried out in complete medium with exosome-free FBS (EXO-FBS-50A, SBI, USA) for $48 \mathrm{~h}$ to obtain at least $200 \mathrm{~mL}$ cell culture supernatant.

Exo were isolated from the supernatant of MSCs by ultracentrifugation. Briefly, the supernatant was centrifuged at $500 \mathrm{~g}$ for $5 \mathrm{~min}$ and at $2000 \mathrm{~g}$ for $30 \mathrm{~min}$ at $4^{\circ} \mathrm{C}$, and mixed with the equivalent volume of pre-cooled 16\% PEG600 (J43968, Jinhui, Shanghai, China). The mixed solution was centrifuged at 10,000 $\mathrm{g}$ (E5810R, Eppendorf, Germany) for $60 \mathrm{~min}$ at $4^{\circ} \mathrm{C}$ to remove cells and debris. In this way, the crude Exo were obtained, and were purified by centrifugation at $100,000 \mathrm{~g}$ for $70 \mathrm{~min}$. After resuspension in PBS (LM0221A, Lianman Shanghai, China), the resulting products were purified Exo. Finally, $400 \mu \mathrm{L}$ Exo solution (concentration at $403.1 \mathrm{ng} / \mu \mathrm{L}$ ) was obtained and placed at $-80^{\circ} \mathrm{C}$.

\section{Design and synthesis of Cy3-siRNA targeting Ctgf gene}

The Ctgf gene sequence (sequence number: ID: 64032) in Genbank database was utilized to match the target sequence. ${ }^{24}$ When designing siRNA, we didn't analyze the non-coding regions at 5 ' and 3 ' ends to obtain sequences (Table 1). However, target gene sequences with GC content ranging from $40 \%$ to $55 \%$ were selected as potentially preferable sequences. A program called BLAST was used to search the sequences in Genbank Expressed Sequence Label (EST) database. The selected sequences were compared on the basis of the corresponding genome library to exclude homologous sequences from other coding ones for determining their specific sequences thereby. To provide the sequence of target gene fragment for further synthesis by Shanghai Meixuan Biological Company (China), three pairs of target gene siRNAs corresponding to different regions were designed and labeled by fluorescent dye Cy3.

\section{Preliminarily analysis for the inhibition efficiency of RNAi}

The target gene-Ctgf fusion transcript reporter system constructed above aimed to explore the inhibition efficiency of the three siRNAs. ${ }^{35}$ HEK293T cells (Cytobank of Saibao, Shanghai, China) were seeded in 6well plates $\left(6 \times 10^{5}\right.$ cells per well) and grown overnight at $37^{\circ} \mathrm{C}$ with $5 \% \mathrm{CO}_{2}$. SiRNA-1, 2 and 3 of $1 \mu \mathrm{g}$ were cotransfected into HEK293T cells using Lipofectamine ${ }^{\mathrm{TM}} 3000$ (Thermo, Shanghai, China). The HEK293T cells without transfection were used as a control. After cotransfection, the expression of Ctgf gene in HEK293T cells was detected at $72 \mathrm{~h}$ by fluorescence microscope (IX71; Olympus, Tokyo, Japan) to preliminarily analyze the inhibition efficiency of RNAi. At the same time, the total RNA of transfected cells was extracted and Ctgf-related mRNAs were analyzed by qRT-PCR (All sequences of primer pairs used were listed in the section of qRT-PCR).

\section{Exosome staining}

According to one research, ${ }^{36}$ the stored Exo were taken out from the fridge and melted. After complete melting, $200 \mathrm{~mL}$ Diluent C (B8003S, Bomez, USA) was added to an EP tube, labeled as A tube, and carefully mixed. Then $300 \mu \mathrm{L}$ Diluent $\mathrm{C}$ and $4 \mu \mathrm{L}$ PKH67 dye (BB-441112, BestBio, Shanghai, China) were added to another EP tube ( $1.5 \mathrm{~mL})$, the $B$ tube, and the dye solution was fully mixed. The solution of $A$ and $B$ tubes was mixed and incubated at room temperature for 5 min. The reaction between Exo and dye 
solution was terminated for 1 min by adding $600 \mu \mathrm{L} 1 \%$ bovine serum albumin (BSA). Then the volume of the mixture was added to $20 \mathrm{~mL}$ and centrifuged for $60 \mathrm{~min}$ at $120,000 \mathrm{~g}$. When the supernatant was discarded, the remaining part was suspended by PBS. The sedimentation was PKH67-Exo.

\section{The loading efficiency of siRNA}

As was mentioned earlier, ${ }^{28}$ we intended to load siRNA into the PKH67-Exo by electroporation using the EC630 electroporation system (BTX, USA). PKH67-Exo was diluted to $0.5 \mathrm{mg} / \mathrm{mL}$ in siRNA electroporation buffer (BTX, USA). Target gene Cy3-siRNA and fluorescently-labeled control siRNA were added at a final concentration of $100 \mathrm{nM}$. The mixtures were transferred into ice cold $0.4-\mathrm{cm}$ cuvettes and electroporated under 8 different conditions, involving changes in voltag, resistance and capacitance (Table 2) with a pulse time of 10-15 ms. Electrical perforation was performed once in each condition. Samples were kept on ice for at least $15 \mathrm{~min}$ before and after an electroporation pulse. The post-electroporation samples were centrifuged at $8,000 \mathrm{~g}$ for $5 \mathrm{~min}$ for pellet aggregates. Cy3-siRNA without Exo was also electroporated to serve as control. Finally, after exosome extraction from electroporation buffer and siRNA extracts, reverse transcription and qPCR detection were performed, and double fluorescent labeled Exo-siRNA was obtained.

\section{Quantitative determination of siRNA-loading capacity in Exo}

Loading capacity: To determine siRNA-loading capacity, the 32P-labeled siRNA was adopted as a tracer using the optimal silencing efficiency which had been screened out in the previous process. The reaction conditions for electroporation were described above. After terminating the reaction, Exo were collected by precipitating with ExoQuick-TC reagent (System Biosciences, Palo Alto, CA, USA) on ice for $1 \mathrm{~h}$ followed by centrifugation at $13,000 \mathrm{Xg}$ for $5 \mathrm{~min}$ and the pellet was resuspended in PBS. The supernatant was collected separately. Radioactivity in the pellet and supernatant was determined by a known volume to a PEl-cellulose layer and quantitated by Packard InstantImager. The siRNA-loading capacity was calculated by the aforesaid formula.

SiRNA y stability siRNA: Exo loaded with 5'-32P-labeled siRNA were incubated with various concentrations of RNase A $\left(0.01 \mu \mathrm{g} / \mathrm{mL}\right.$, Sigma, USA). After incubation at $37^{\circ} \mathrm{C}$ for $45 \mathrm{~min}$, naked siRNA was used as control. Quantitative analysis of undegraded siRNA was performed by RT-PCR.

\section{Co-culture of Exo-siRNA with astrocytes, DRGs and macrophages in $\mathrm{SCl}$ environment}

\section{Co-culture with astrocytes}

Rat astrocytes (ATCC, Chinese Academy of Sciences cell bank) were inoculated on 12-well plate, with $5 \times$ $10^{4}$ cell/well. The $2 \mathrm{~mL}$ DMEM medium (Thermo, 11965-092) encompassed $10 \%$ fetal bovine serum (Gibco, 10099-141), $100 \mu \mathrm{g} / \mathrm{mL}$ streptomycin (Gibco) and $100 \mathrm{U} / \mathrm{mL}$ penicillin (Gibco). When astrocytes fused to about $90 \%$, the $\mathrm{SCl}$ environment was simulated by $100 \mu \mathrm{L}$ LPS $(10 \mu \mathrm{g} / \mathrm{mL}$, Jinhui, China) and hypoxia $\left(2 \% \mathrm{O}_{2}\right)$ for 24 hours. Cell grouping $(n=3)$ : $\mathrm{SCl}$ group was treated with $50 \mu \mathrm{L}$ PBS; siRNA group 
was incubated by $2 \mu \mathrm{L}$ lipo3000 (Thermo, L3000015) and $50 \mathrm{nM}$ Cy3-siRNA, as negative control (NC); Exo group was added with $50 \mu \mathrm{L}$ Exo; and Exo-siRNA group contained $50 \mu \mathrm{L}$ Exo-siRNA. RNAs were extracted $48 \mathrm{~h}$ after incubation for further quantitative RT-PCR analysis. Proteins were also extracted for WB.

\section{Co-culture with DRGs}

Rat spinal DRGs (ATCC, Chinese Academy of Sciences cell bank) were sampled. Cell culture, seedboard, grouping and administration were the same as those of the astrocytes which were mentioned above. It was noted that cells of the control group were not treated with LPS/hypoxia. After the 24-h administration, DRG neurons were washed with PBS twice and stained in situ. Each 12-well plate was rearranged with $200 \mu \mathrm{L}$ Calcein-AM/PI double staining reagent (Saibaikang, HR0444, China) and the neurons were incubated at $37^{\circ} \mathrm{C}$ for $25 \mathrm{~min}$. Cells of all groups were observed by fluorescence microscopy (Olympus, IX70, Japan) with the $490 \mathrm{~nm}$ excitation wavelength. Yellow/green fluorescence indicated living cells, and red fluorescence marked dead cells. Cell viability was detected by CCK-8. Cell suspension was collected and inoculated into a 96 -well plate $(100 \mu \mathrm{L}$ per well). Subsequently, $10 \mu \mathrm{L}$ of CCK-8 solution (Abbkine, KTC011001) was added to each well and incubated for $2.5 \mathrm{~h}$. The absorbance (OD) at $450 \mathrm{~nm}$ was measured by an enzyme immunoassay microplate reader.

\section{Co-culture with macrophages}

Rats NR8383 macrophages (ATCC, Chinese Academy of Sciences cell bank) were obtained. Cell culture, seed plate, grouping and administration were the same as those of astrocytes. After 48-h treatment, RNAs were extracted for RT-PCR analysis and an ELISA assay was adopted to detect expressions of various inflammatory factors using the following kits: rat IL-1 $\beta$ ELISA kit (Cusabio, China), rat TNF- $a$ ELISA kit (Cusabio, China), rat IL-6 ELISA kit (Cusabio, China), rat IL-4 ELISA kit (Cusabio, China), rat IL-10 ELISA kit (Cusabio, China) and rat VEGF ELISA kit (Cusabio, China). Cell supernatant was collected and centrifuged for $20 \mathrm{~min}$ at $1,000 \mathrm{~g}$. The OD at $450 \mathrm{~nm}$ was measured by an ELISA reader.

\section{Establishment, grouping and tail-vein administration of SCl rat models}

The animal experiments in this study were reviewed and approved by the ethics committee of Affiliated Zhongshan Hospital of Dalian University. One hundred SD female rats (200-250 g, 10 weeks old) were randomly divided into 5 groups: SCI group (PBS treatment), Sham group (PBS treatment), siRNA group (siRNA treatment), Exo group (pure exosome treatment) and Exo-siRNA group (Exo-siRNA treatment) with 20 rats in each group. Rats were anaesthetized with sodium pentobarbital (Sigma, St. Louis Mo, USA). An incision of $2 \mathrm{~cm}$ was made at T10 level to expose skeletal muscles of spinal cord in sterile environment. Lateral vertebral muscles were separated from the bone margin of articular process. Spinous processes and laminae were removed and T10 was exposed as an impact zone. Except for the Sham group, receiving a sham operation, rats of the rest groups were hit on the T10 segment using a spinal cord impactor (PSI, IH-0400, USA), with metal rod weight of $30 \mathrm{~g}$ and height of $50 \mathrm{~mm}$. After that, bilateral hind limbs were involuntarily twitching and tails swinging in all animals, indicating that the injury corresponded to the standard of a SCI model. After the suture of muscle layer and skin, rats were allowed 
to recover in heat preservation chambers. Postoperative nursing of SCI rats mainly encompassed daily artificial bladder emptying and restoration of bladder reflex activity. Tail-vein administration was performed every $24 \mathrm{~h}$ for 5 consecutive days, and the dosage was as follows: SCl group and Sham group were administered with $150 \mu \mathrm{L}$ PBS; while siRNA group with $150 \mu \mathrm{L}$ siRNA $(0.2 \mathrm{nmol})$, Exo group with 150 $\mu \mathrm{L}$ exosome solution and Exo-siRNA group with $150 \mu \mathrm{L}$ Exo-siRNA solution.

\section{Quantitative qRT-PCR}

A RNA extraction kit (Solarbio, China) was used to extract total RNA from various cells and spinal cord tissues. SiRNAs from Exo or cells were extracted by a miRNA extraction kit (Solarbio, China). Target mRNA or siRNA was used as template, and the total RNA reverse-transcribed into CDNA using a reverse transcription kit (Solarbio, China). The volume of the whole reaction system was $15 \mu \mathrm{L}$, which was maintained at $16^{\circ} \mathrm{C}$ for $30 \mathrm{~min}, 42^{\circ} \mathrm{C}$ for $30 \mathrm{~min}$ and $85^{\circ} \mathrm{C}$ for $5 \mathrm{~min}$. A universal PCR kit (Solarbio China) was used for PCR amplification using CDNA as template. Quantitative qRT-PCR was performed under the following amplification conditions: $95^{\circ} \mathrm{C}$ for $20 \mathrm{~s}$, followed by 40 cycles of denaturation at 95 ${ }^{\circ} \mathrm{C}$ for $3 \mathrm{~s}$ with extension at $60{ }^{\circ} \mathrm{C}$ for $30 \mathrm{~s}$. The reaction repeated three times. Gapdh was adopted as an internal reference gene for relative quantification by the $2^{-\Delta \Delta C t}$ method.

\section{Western blotting}

Total protein of Exo, cells or spinal cord tissues was extracted using RIPA buffer (Jinhui, Shanghai, China) and protein concentration was evaluated with a BCA assay (Jinhui, Shanghai, China) according to the manufacturer's protocol. Proteins ( $50 \mu \mathrm{g}$ per lane) were separated by $10 \%$ SDS-PAGE and transferred to polyvinylidene difluoride membranes. The membranes were blocked using $5 \%$ non-fat dry milk in TBST at $37^{\circ} \mathrm{C}$ for $1 \mathrm{~h}$ and incubated with primary antibodies at $4^{\circ} \mathrm{C}$ overnight. All primary antibodies included CD9, CD63, GAPDH, CTGF, GFAP, vimentin, fibronectin, laminin, HGF, VEGF, BDNF, TGF- $\beta 1$, Bcl-2 and Caspase-3 (Abcam, UK). Subsequent to washing with PBST for $20 \mathrm{~min}$, membranes were incubated with anti-rabbit horseradish peroxidase-conjugated secondary antibody (Abcam, UK) at $37^{\circ} \mathrm{C}$ for $1 \mathrm{~h}$. Protein blanks were visualized with an enhanced chemiluminescence kit (Promo Cell, Germany).

\section{Immunofluorescence Analysis}

Rat spinal cords were sectioned on a cryostat set at 20- $\mu \mathrm{m}$ intervals. Sections were permeabilized in $0.5 \%$ Tween solution, blocked with $5 \%$ bovine serum, and then incubated with rabbit GFAP antibody (1:100, Abcam, UK) and mouse neurofilament NF-H antibody (1:1000, Biorbyt, UK) at $4^{\circ} \mathrm{C}$ overnight. After that, sections were incubated with goat anti-rabbit $\lg G(H+L)$ secondary antibody $(1: 300$, Invitrogen), and DAPI (1:1000) for $1 \mathrm{~h}$, mounted with coverslips, and confocal fluorescence microscope (Leica Microsystems DM i8) was performed to obtain images.

\section{Masson staining}


After 28 days, the spinal cords of rats were harvested and sectioned $(2 \mathrm{~mm})$ in cross section. After dewaxing, the samples were washed with distilled water three times. Then they were stained with nuclear dye for 2 min, destained with 1\% hydrochloric acid alcohol (75\% alcohol : concentrated hydrochloric acid = 99:1) for $1 \mathrm{~s}$, blue in running tap prior to cytoplasmatic staining for 30-60 s. Finally, color separation solution for 1-3 min and the sections were transferred to aniline blue solution and stained for $5 \mathrm{~min}$. The slides were dehydrated and the tissue was mounted with a coverslip. The accomplished slides of spinal cords were observed under a confocal microscope (Leica Microsystems SD AF).

\section{BBB locomotor scale}

Rat locomotor recovery was estimated by the BBB locomotor rating scale, ranging from 0 point (no motor activity) to 21 points (normal locomotion). Two independent investigators blinded to the treatment assessed the locomotor activity with corresponding scores at 1, 7, 14, 21, 28, 35, 42 and 48 days after modeling.

\section{Statistical analysis}

Statistical analyses were performed using MATLAB/GraphPad Prism software. Student $t$ test was utilized for two group comparison. One-way ANOVA was adopted to compare the differences between groups while multiple comparisons were performed by Tukey's post hoc test (Graphpad Prism 8.0). $P$ values of $<0.05$ indicated statistical significance. Significance levels were represented as ${ }^{*} p<0.05$ and ${ }^{*} p<0.01$.

\section{Conclusion}

This study demonstrates that Exo-siRNA presents high targeting ability in vivo and its feasibility for repairing the injured spinal cord. Exo act not only as efficient carriers for biologically active molecules, but prominent facilitators for resisting neuronal apoptosis, stimulating the secretion of neuroprotective factors and anti-inflammatory factors and ameliorating microenvironment of impaired spinal cord. These effects mainly attribute to the integrated function of various RNAs, proteins and other messenger molecules contained in Exo, which requires further precise analysis and clarification. However, exosome, a non-invasive, rapid and cell-free deliverer with low specificity, shows broad prospects and will inspire more effective methods for the treatment of SCl.

\section{Declarations}

\section{Author information}

Corresponding Author *E-mail: yuxiaobing1976@hotmail.com

\section{Availability of data and materials}

All data generated or analyzed during this study are included in this published article. 


\section{Authors' contributions}

Conceptualization: Wei Huang, Mingjia Qu囚Lu Li.

Investigation: Ruimeng Duan, Depeng Shang.Lu Li

Methodology: Meng Zhang,Yashuai Yuan.

Resources: Xiaobing Yu, Mingjia Qu.

Supervision: Xiaobing Yu,Wei Huang,Lu Li.

Writing - original draft: Wei Huang, Mingjia Qu,Xiaobing Yu.

Writing - review \& editing: Wei Huang, Xiaobing Yu.

\section{Funding}

There is no fund support here.

\section{Ethics approval and consent to participate}

This work was supported by Affiliated Zhongshan Hospital of Dalian University according to Laboratory Animal Welfare and Ethical review.

\section{Consent for publication}

All the authors have approved the manuscript and agree with submission to

your esteemed journal.

\section{Competing interests}

The authors declare that they have no competing interests.

\section{References}

1. FE, P.; neurology, N. H. J. E., Serotonergic mechanisms in spinal cord injury. 2019,318 (undefined), 174-191.

2. FA, R.; JE, G.; JD, R.; AJ, H.; J, L.; medicine, D. J. J. A. o. p.; rehabilitation, Medical rehabilitation after natural disasters: why, when, and how? 2012,93 (10), 1875-81.

3. P, A.; GJ, D.; BJ, H.; JR, P.; neuroscience, T. W. J. N., Cell transplantation therapy for spinal cord injury. 2017,20(5), 637-647.

4. H, S.-P.; R, R.; J, M.; L, R.; CR, C.; S, H.; N, A.; sciences, A. M. J. L., Intra-bladder wall transplantation of bone marrow mesenchymal stem cells improved urinary bladder dysfunction following spinal cord 
injury. 2019,221 (undefined), 20-28.

5. JH, H.; XM, Y.; Y, X.; CC, X.; X, L.; FB, Y.; Y, C.; neurotrauma, L. F. J. J. o., Systemic Administration of Exosomes Released from Mesenchymal Stromal Cells Attenuates Apoptosis, Inflammation, and Promotes Angiogenesis after Spinal Cord Injury in Rats. 2017,34 (24), 3388-3396.

6. N, P.; O, B.; R, S.; S, B.; A, A.; T, S.; U, A.; R, P.; letters, O. D. J. N., Golden Exosomes Selectively Target Brain Pathologies in Neurodegenerative and Neurodevelopmental Disorders. 2019,19 (6), 3422-3431.

7. G, M.; CV, C.; EJ, H.; JR, P.; LI, Z.; N, M.; TE, P.; DE, I.; CC, D.; nano, M. M. J. A., Tumor-Derived Extracellular Vesicles Breach the Intact Blood-Brain Barrier Transcytosis. 2019, undefined (undefined), undefined.

8. DM, P.; biochemistry, G. S. J. A. r. o., Exosomes. 2019,88 (undefined), 487-514.

9. M, T.; Cell, T. C. J., Communication by Extracellular Vesicles: Where We Are and Where We Need to Go. 2016,164 (6), 1226-1232.

10. S, P.; Cell, S. J. J., Explicating Exosomes: Reclassifying the Rising Stars of Intercellular Communication. 2019,177 (2), 225-227.

11. DK, J.; AM, F.; JL, F.; JN, H.; Q, Z.; LJ, Z.; DC, L.; J, P.; Q, L.; R, E.; WH, F.; JG, P.; LH, R.; DT, B.; Cell, C. R. J., Reassessment of Exosome Composition. 2019,177(2), 428-445.e18.

12. JK, W.; neurology, S. R. J. C. o. i., Traumatic brain injury: recent advances in plasticity and regeneration. 2015,28 (6), 565-73.

13. Y, Y.; Y, Y.; X, S.; J, H.; W, B.; neuroscience, H. X. J. F. i. C., MSCs-Derived Exosomes and Neuroinflammation, Neurogenesis and Therapy of Traumatic Brain Injury. 2017,11 (undefined), 55.

14. Y, Z.; M, C.; Y, M.; M, K.; H, X.; A, M.; neurosurgery, X. Y. J. J. O., Effect of exosomes derived from multipluripotent mesenchymal stromal cells on functional recovery and neurovascular plasticity in rats after traumatic brain injury. 2015,122 (4), 856-67.

15. ZG, Z.; B, B.; Neurology, C. M. J. N. r., Exosomes - beyond stem cells for restorative therapy in stroke and neurological injury. 2019,15(4), 193-203.

16. Sun, G.; Li, G.; Li, D.; Huang, W.; Zhang, R.; Zhang, H.; Duan, Y.; Wang, B. J. M. S.; C, E., hucMSC derived exosomes promote functional recovery in spinal cord injury mice via attenuating inflammation. 2018,89.

17. M, M.; K, R.; transplantation, S. E. J. B. m., Mesenchymal stem cell-derived exosomes for clinical use. 2019,54 (null), 789-792.

18. X, W.; H, C.; pharmacology, W. S. J. E. j. o., CTGF: A potential therapeutic target for Bronchopulmonary dysplasia. 2019,860 (undefined), 172588.

19. Q, Y.; medicine, L. H. J. A. i. e.; biology, Connective Tissue Growth Factor and Renal Fibrosis. 2019,1165 (undefined), 365-380.

20. S, O.; H, T.; SU, K.; Cellular, S. J. J.; neurobiology, m., Gene expression profiling of human neural progenitor cells following the serum-induced astrocyte differentiation. 2009,29 (3), 423-38. 
21. AP, T.; PM, W.; reviews, S. J. J. P., The Biology of Regeneration Failure and Success After Spinal Cord Injury. 2018,98 (2), 881-917.

22. MH, M.; C, P.; AL, D.; T, E.; DY, S.; Science, P. K. J., Injury-induced ctgfa directs glial bridging and spinal cord regeneration in zebrafish. 2016,354 (6312), 630-634.

23. Lu, L.; Wang, Y.; Zhang, F.; Chen, M.; Lin, B.; Duan, X.; Cao, M.; Zheng, C.; Mao, J.; Shuai, X. J. A. F. M., MRI-Visible siRNA Nanomedicine Directing Neuronal Differentiation of Neural Stem Cells in Stroke.

24. Y, W.; QJ, K.; JC, S.; Y, Y.; HB, W.; Q, Z.; Society, S. J. J. T. s. j. o. j. o. t. N. A. S., Lentivirus-mediated silencing of the CTGF gene suppresses the formation of glial scar tissue in a rat model of spinal cord injury. 2018,18 (1), 164-172.

25. Danilov, C. A.; Steward, O. J. E. N., Conditional genetic deletion of PTEN after a spinal cord injury enhances regenerative growth of CST axons and motor function recovery in mice. 2015,266, 147160.

26. EA, G.; MM, B.; neurology, S. O. J. E., Long-term consequences of conditional genetic deletion of PTEN in the sensorimotor cortex of neonatal mice. 2016,279 (undefined), 27-39.

27. S, G.; N, P.; O, B.; S, B.-S.; A, S.; I, M.; R, P.; D, O.; nano, L. S. J. A., Intranasal Delivery of Mesenchymal Stem Cell Derived Exosomes Loaded with Phosphatase and Tensin Homolog siRNA Repairs Complete Spinal Cord Injury. 2019, undefined (undefined), undefined.

28. FN, F.; L, X.; JoVE, A.-J. K. J. J. O. v. e., Preparation of Exosomes for siRNA Delivery to Cancer Cells. 2018, undefined (142), undefined.

29. F, A.; R, M.; J, J.; AK, A.; AH, K.; SA, W.; letters, G. R. J. C., Milk exosomes - Natural nanoparticles for siRNA delivery. 2019,449 (undefined), 186-195.

30. HY, K.; H, K.; MJ, J.; J, K.; JK, Y.; JR, L.; M, K.; YW, C.; SY, S.; SP, K.; T, H.; IB, H.; letters, K. B. J. N., Therapeutic Efficacy-Potentiated and Diseased Organ-Targeting Nanovesicles Derived from Mesenchymal Stem Cells for Spinal Cord Injury Treatment. 2018,18 (8), 4965-4975.

31. X, W.; K, C.; X, S.; Y, C.; Z, D.; L, S.; L, G.; P, B.; D, S.; J, F.; X, H.; W, Y.; Glia, R. Y. J., Macrophages in spinal cord injury: phenotypic and functional change from exposure to myelin debris. 2015,63 (4), 635-51.

32. R, S.; O, M.; G, Y.; N, R.; A, L.; J, R.; KW, K.; E, K.; V, K.; P, B.; SA, L.; S, J.; Immunity, S. M. J., Recruitment of beneficial M2 macrophages to injured spinal cord is orchestrated by remote brain choroid plexus. 2013,38 (3), 555-69.

33. W, L.; Y, W.; F, G.; Y, R.; Y, L.; P, T.; Z, Z.; Z, Z.; T, X.; T, J.; S, Y.; G, Y.; J, C.; J, F.; neurotrauma, C. W. J. J. o., Exosomes Derived from Bone Mesenchymal Stem Cells Repair Traumatic Spinal Cord Injury by Suppressing the Activation of A1 Neurotoxic Reactive Astrocytes. 2019,36 (3), 469-484.

34. MB, G.; T, M.; E, C.; CJ, H.; J, G.; C, H.; D, T.; M, R.; M, A.; P, D.; TP, C.; Neuroscience, C. J. J. T. J. o. n. t. o. j. o. t. S. f., Neuronal RAR $\beta$ Signaling Modulates PTEN Activity Directly in Neurons and via Exosome Transfer in Astrocytes to Prevent Glial Scar Formation and Induce Spinal Cord Regeneration. 2015,35 (47), 15731-45.

35. H, Z.; X, L.; Q, W.; F, L.; Z, F.; C, L.; Z, L.; T, C.; T, W.; L, L.; G, N.; X, K.; medicine, F. S. J. R., shRNA against PTEN promotes neurite outgrowth of cortical neurons and functional recovery in spinal cord 
contusion rats. $2015,10(4), 411-29$.

36. B, X.; Y, Z.; XF, D.; J, L.; HX, Z.; JW, B.; Y, Y.; H, H.; research, D. J. J. C., Neurons secrete miR-132containing exosomes to regulate brain vascular integrity. 2017,27(7), 882-897.

\section{Figures}

a

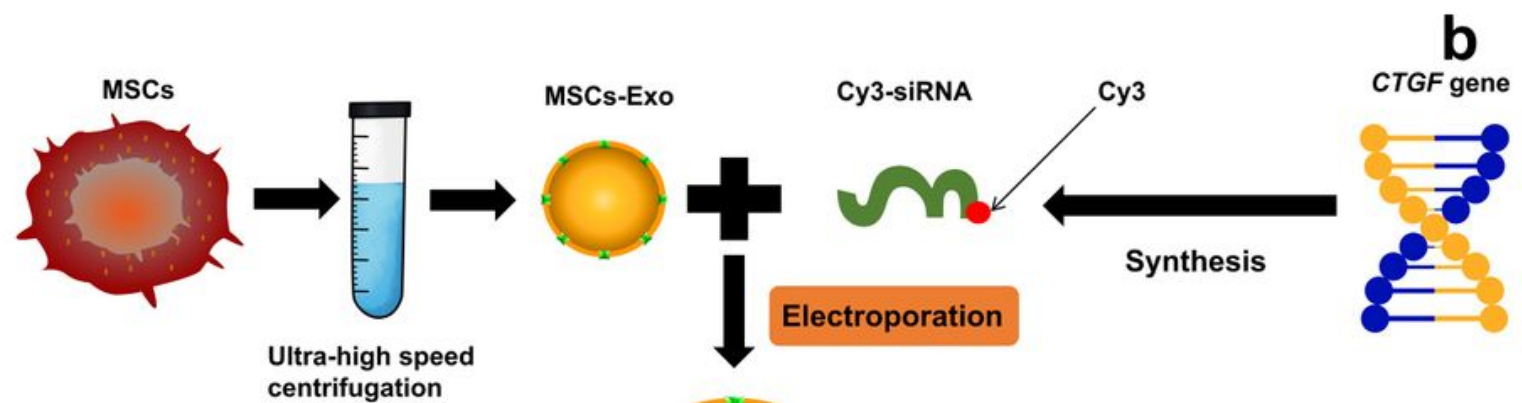

C

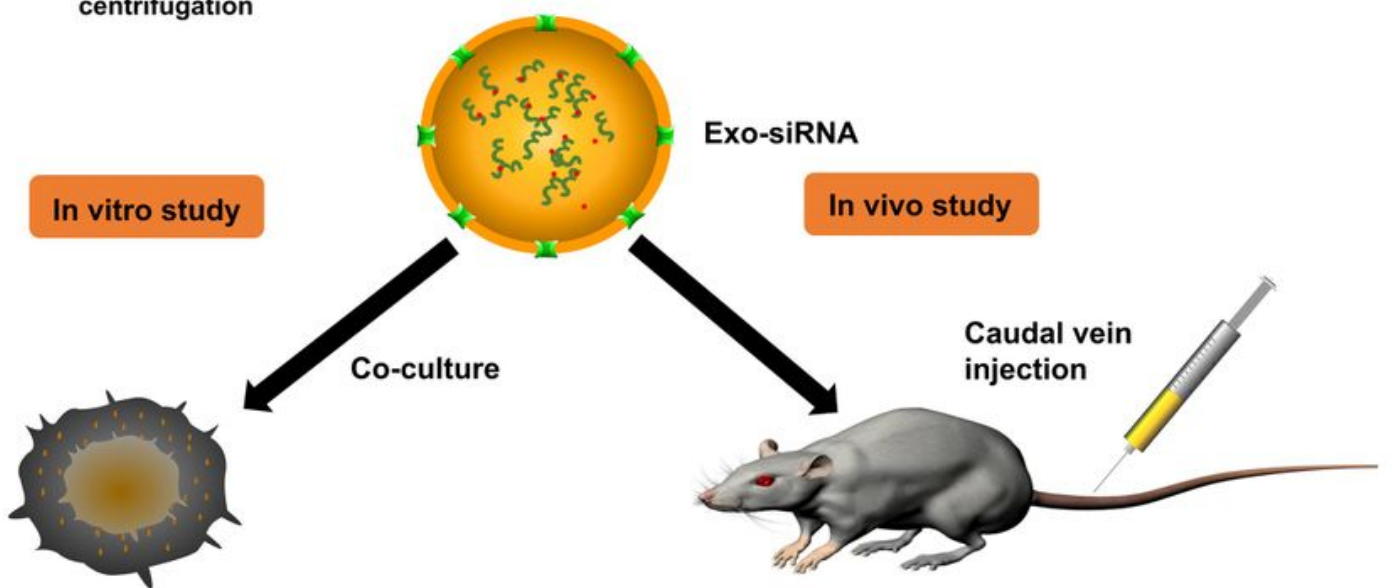

Spinal cord injury-related cells

$\mathrm{SCl}$ rat

d

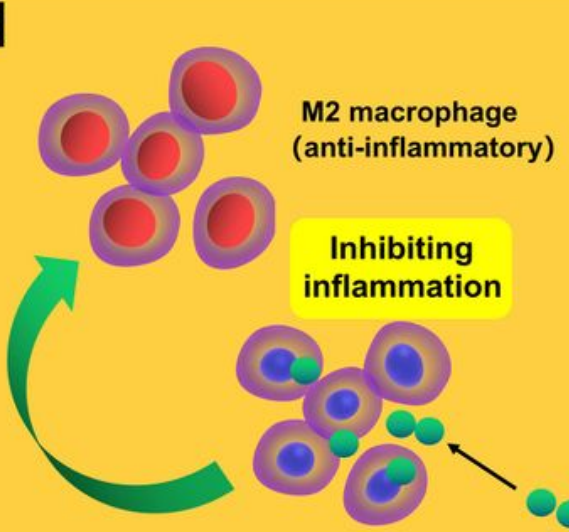

M1 macrophage

(Proinflammatory)

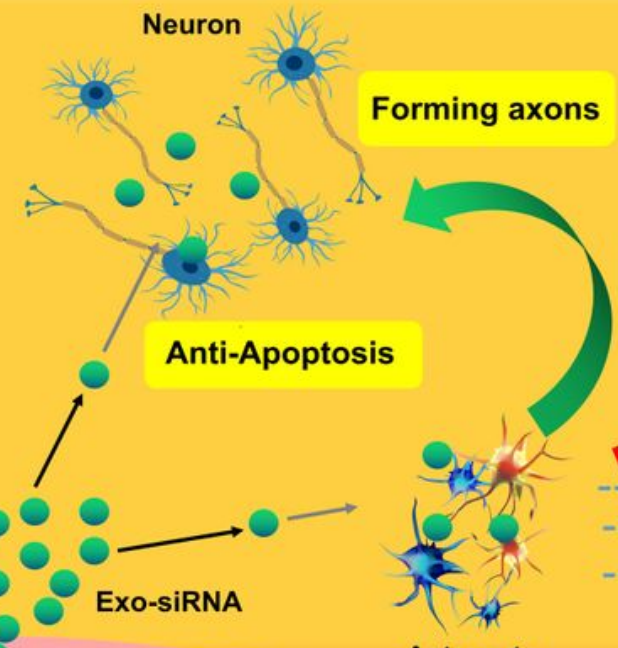

Astrocyte
Spinal cord

Secretion of neurotrophic factors

\section{Blood vessel}


Figure 1

a
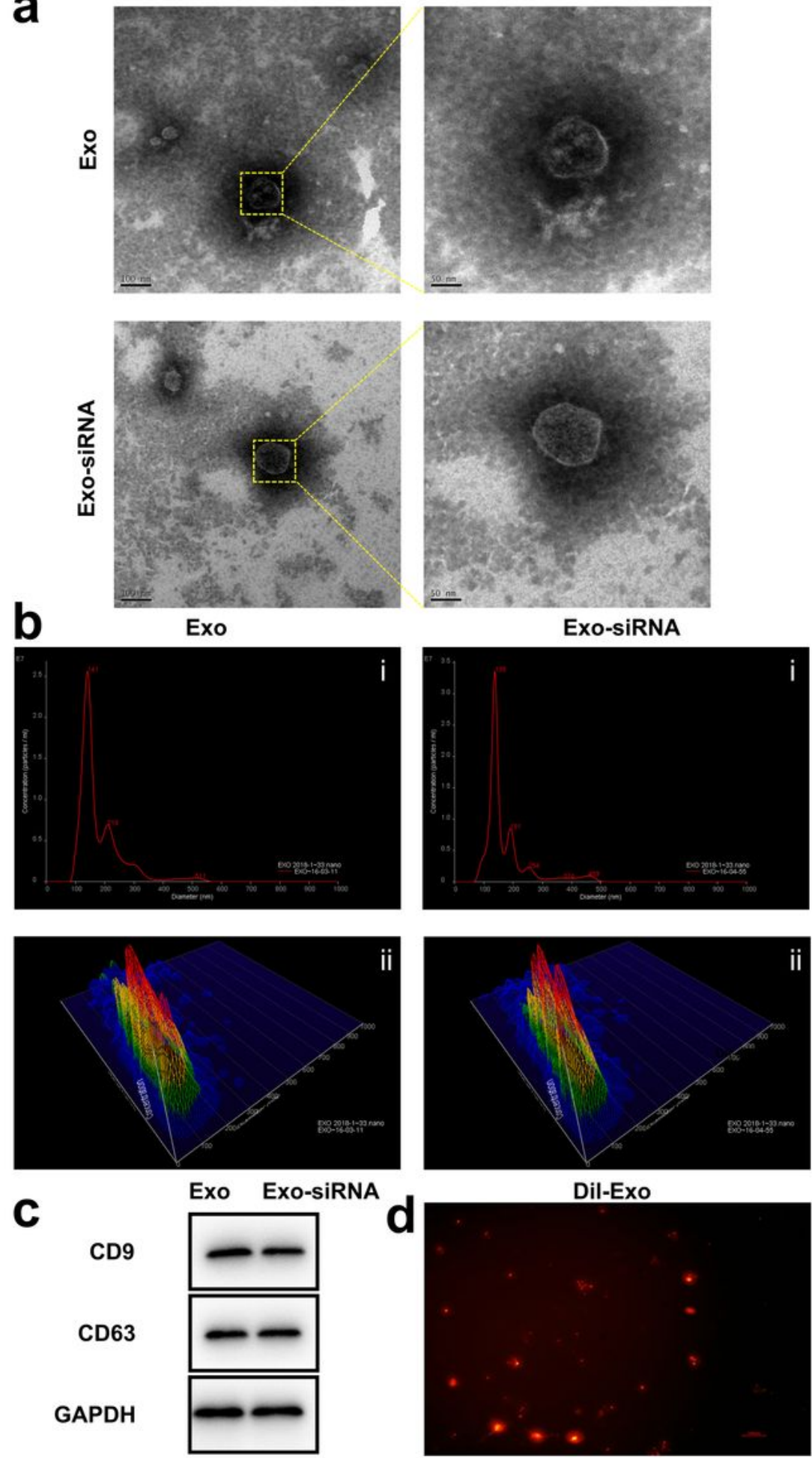

Dil-Exo

Figure 2

Characterization of Exo and Exo-siRNA. (a) TEM revealed the typical exosome structure of Exo and ExosiRNA. (b) NTA analysis of Exo and Exo-siRNA showed the incorporation of siRNA did not affect the 
diameter of Exo. (c) The exosome marker proteins, CD9 and CD63, were analyzed by WB. (d) Exo stained by fluorescence of DilC18 amethylindocarbocyanine were exhibited, scale bar $=100 \mu \mathrm{m}$.

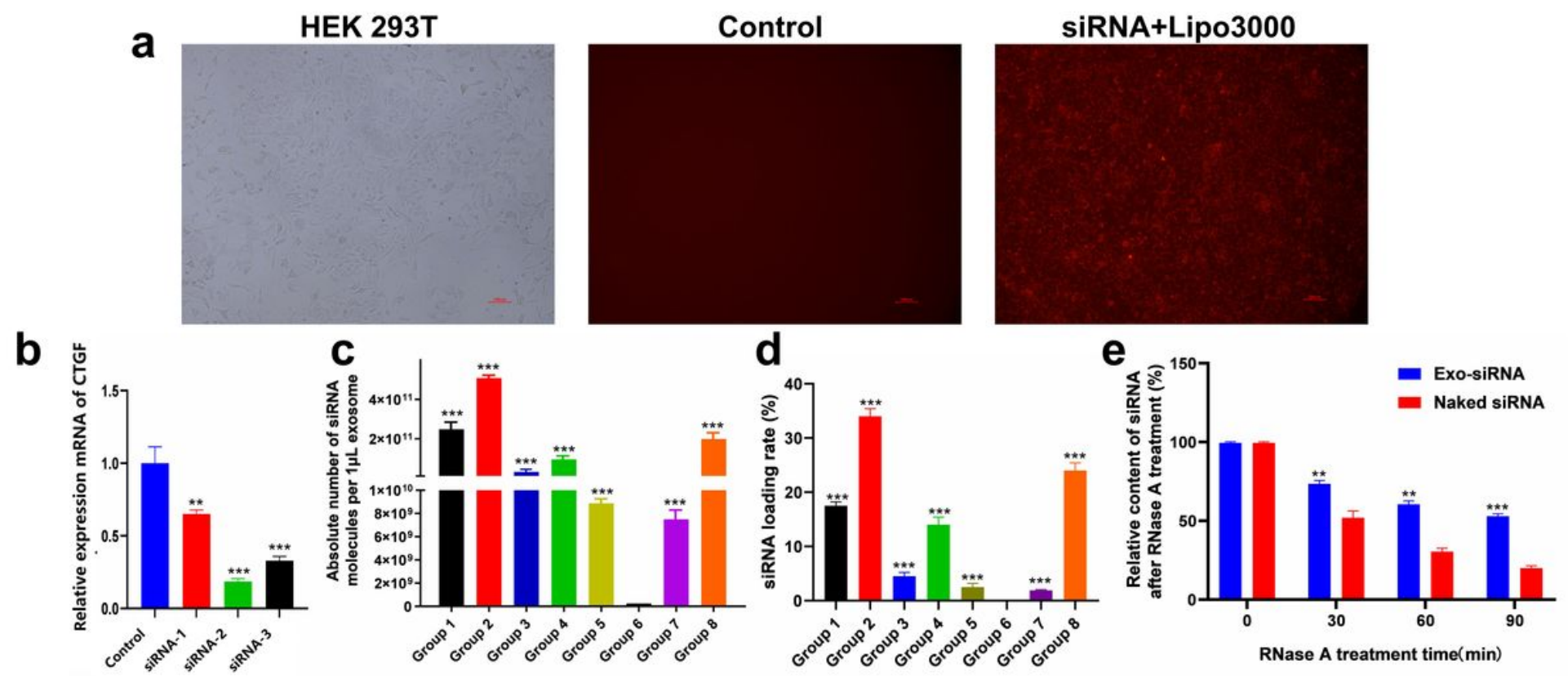

Figure 3 


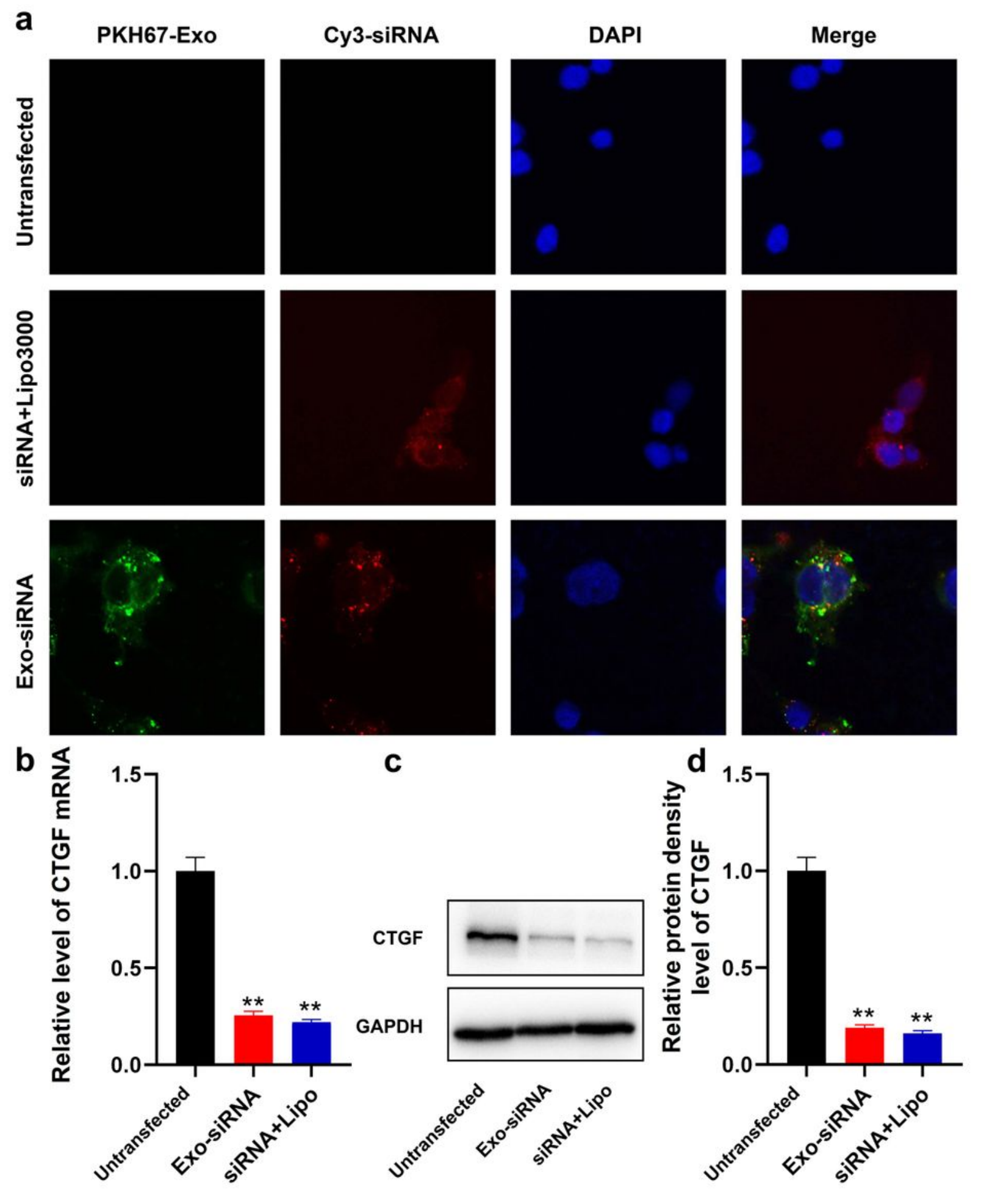

Figure 4 
a
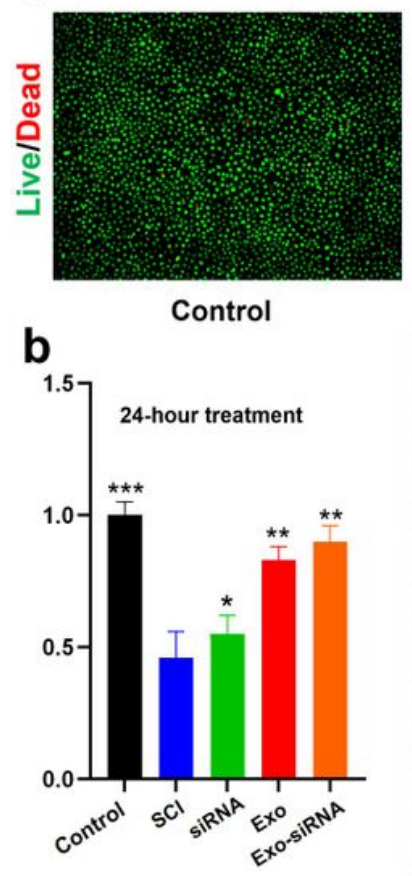

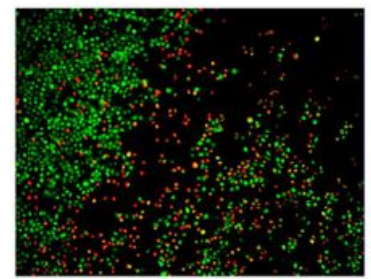

SCl+siRNA

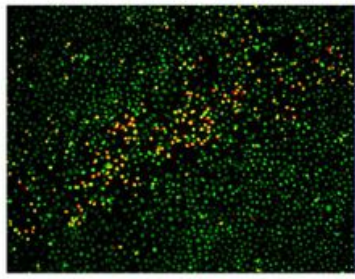

SCl+Exo

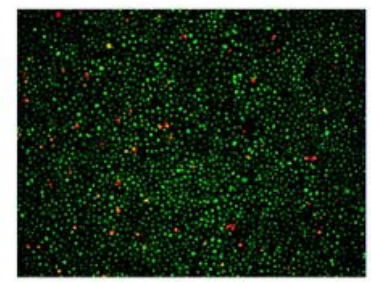

SCl+Exo-SiRNA

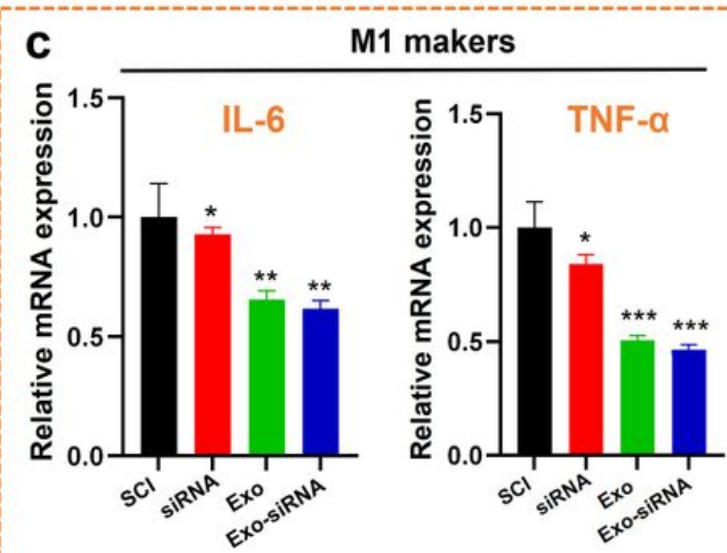

d M2 makers
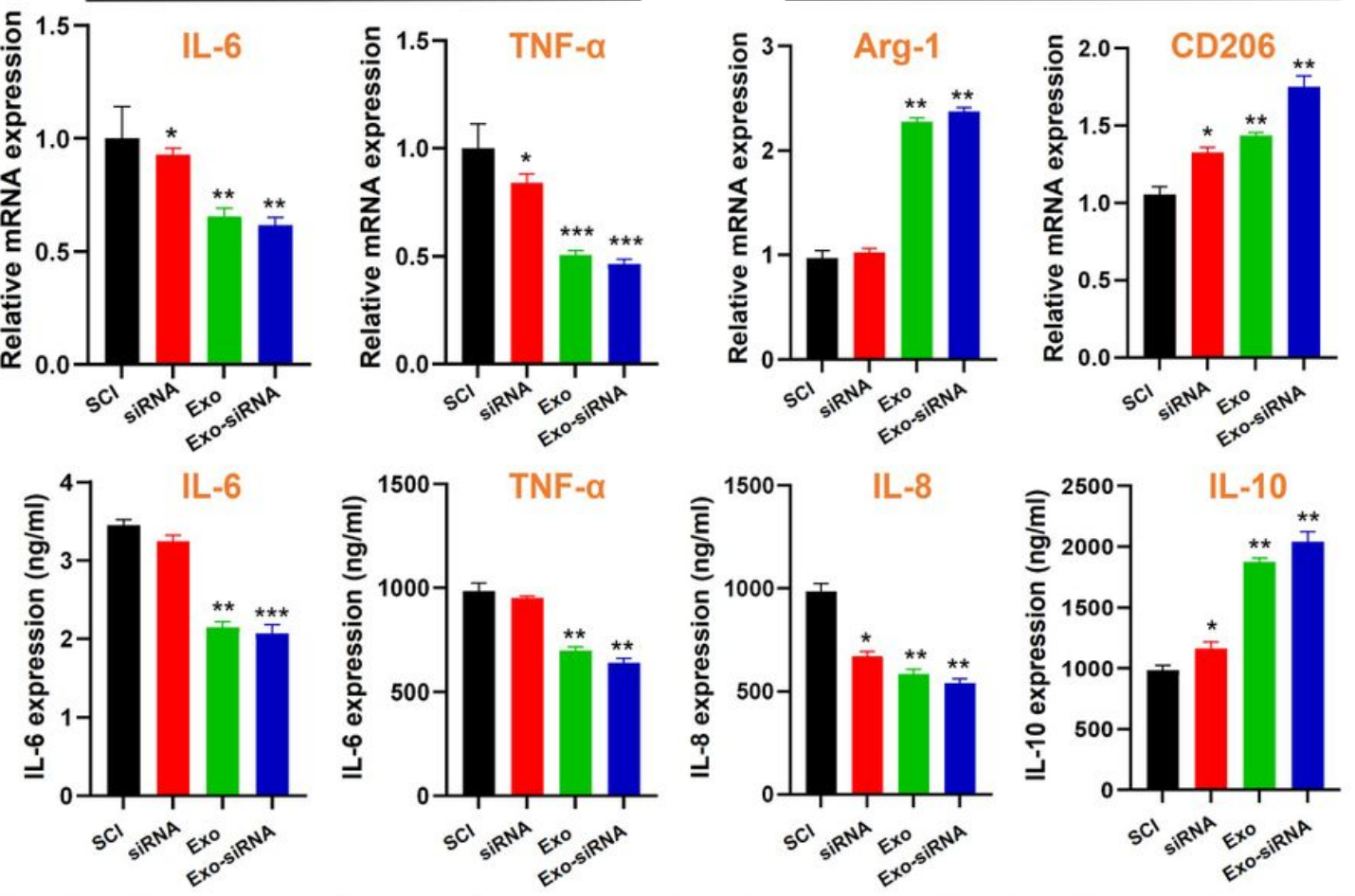

Figure 5 


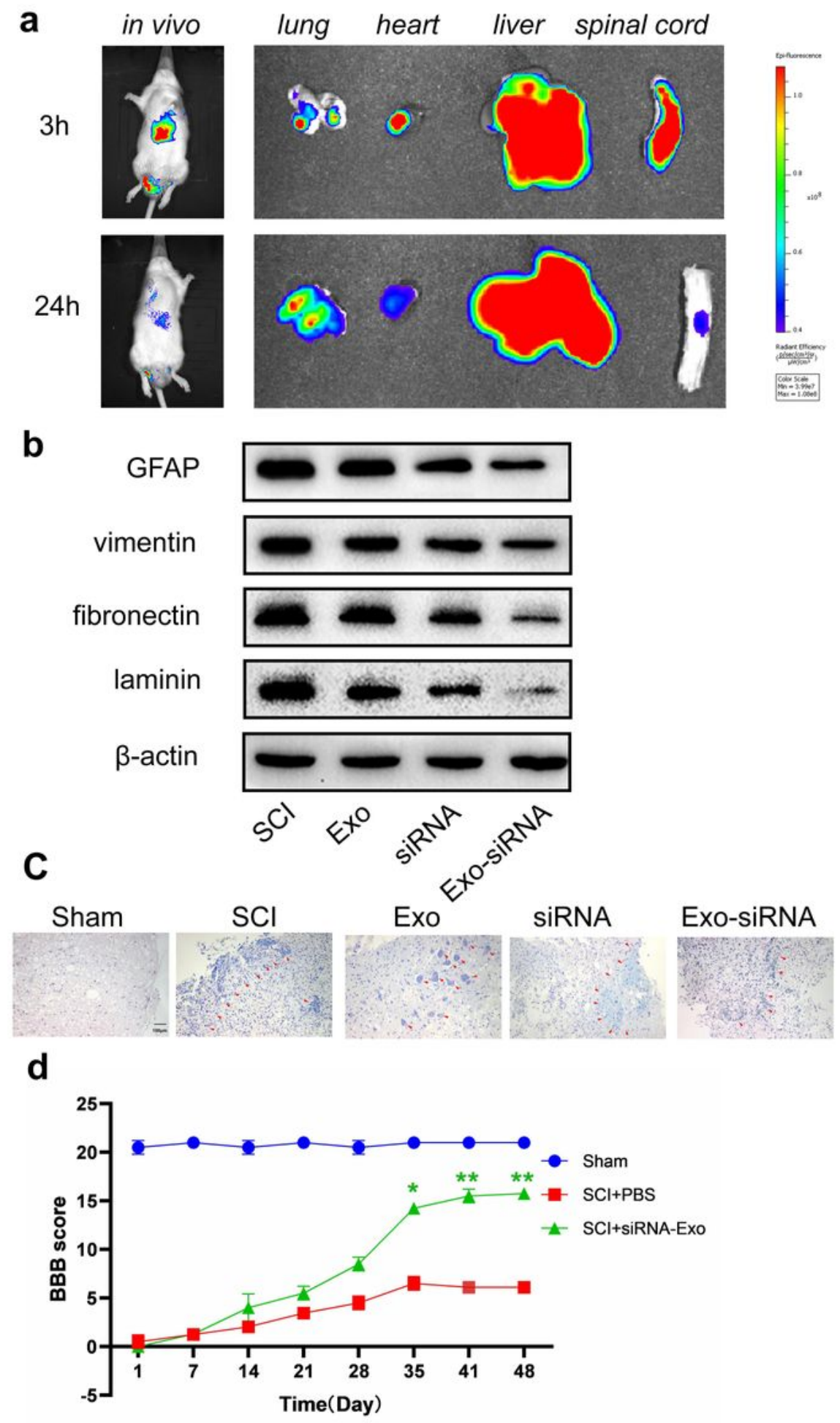

Figure 6 
a

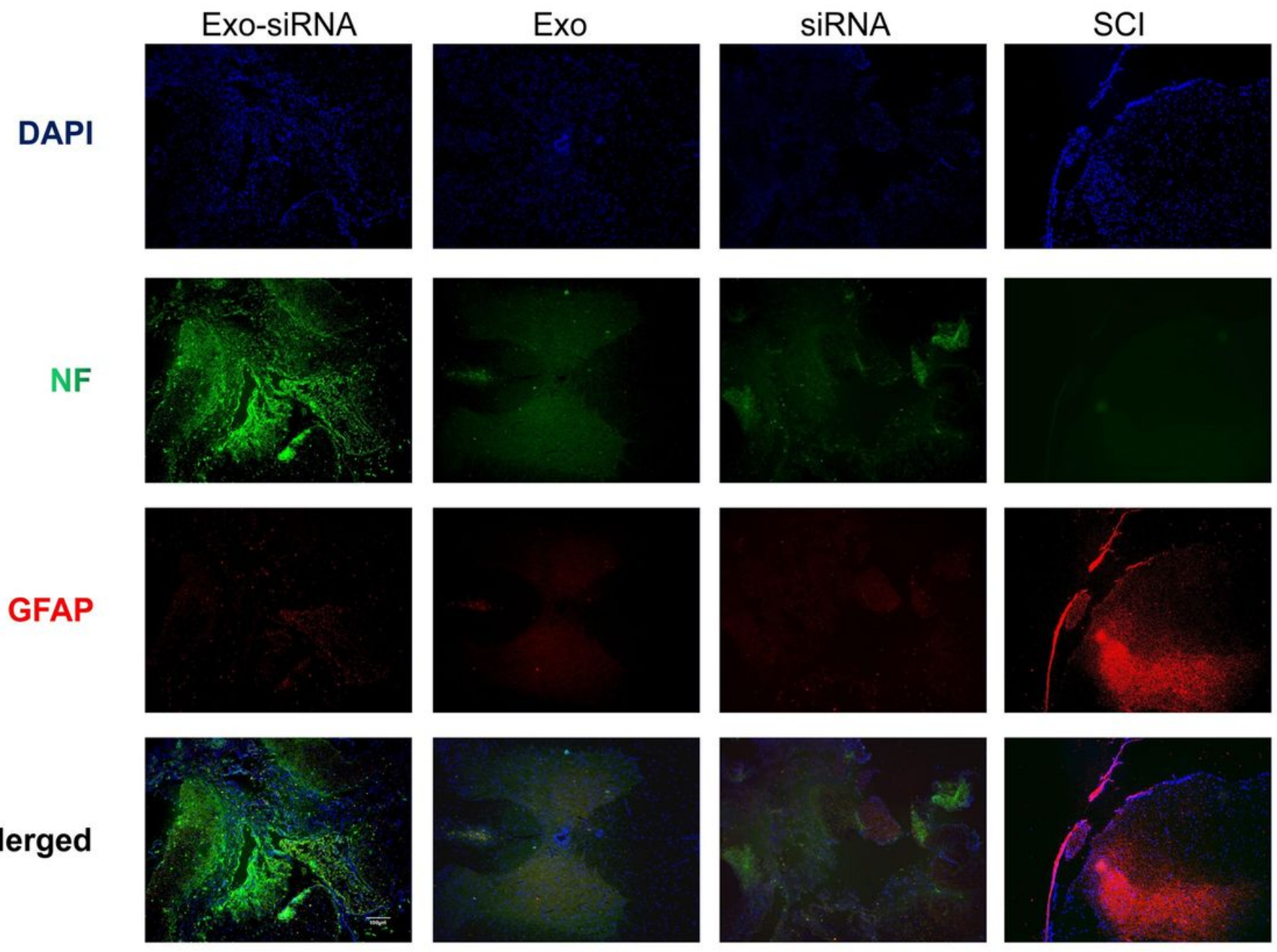

Figure 7 